\title{
Pénuries de médicaments : plus qu'un simple bruit de fond
}

\author{
par Susan K Bowles
}

$L^{2}$ e Journal canadien de la pharmacie hospitalière (JCPH) a publié en 2012 un premier éditorial sur les pénuries de médicaments ${ }^{1}$. Près de sept ans plus tard, le présent numéro du JCPH comprend une étude comparant plusieurs aspects des pénuries de médicaments au Canada et dans quatre pays d'Europe, ce qui nous rappelle que le problème est loin d'être réglé. Videau et collab. ${ }^{2}$ ont remarqué que les cinq lieux à l'étude étaient quotidiennement confrontés à des pénuries de médicaments, principalement en ce qui concerne les produits injectables. De plus, la durée des pénuries pouvait se prolonger : dans l'hôpital canadien à l'étude, la durée médiane était de 32 jours, mais certaines pénuries pouvaient durer plus d'un an, la plus longue ayant été signalée avait d'ailleurs sévi pendant 402 jours ${ }^{2}$. Ces données correspondent à celles émises par Pénuries de médicaments Canada, qui, depuis le début de 2018, estime à environ 300 le nombre de pénuries touchant des vaccins, des antinéoplasiques, des antibiotiques et d'autres médicaments employés régulièrement en milieu hospitalier ${ }^{3}$. En effet, les pénuries de médicaments sont à ce point fréquentes que certains pharmaciens en sont venus à considérer ce problème comme le «bruit de fond » de la pratique de la pharmacie hospitalière d'aujourd'hui (Stephen Shalansky, Providence Healthcare, Services de pharmacie des basses-terres continentales; communication personnelle, 8 novembre 2018).

Les pénuries de médicaments peuvent affecter plusieurs endroits de la chaîne d'approvisionnement. Par exemple, il peut y avoir des pénuries ou une contamination des matériaux de base ou des principes actifs, des problèmes de fabrication liés au contrôle de la qualité, des retards de production et des problèmes de fournisseurs, par exemple quand on ne compte que sur un seul fournisseur ${ }^{4}$. Les services de pharmacie font de leur mieux pour réduire les impacts de ce problème courant, mais il est certain que, malgré ces efforts, les pénuries de médicaments peuvent avoir une influence négative sur la qualité des soins offerts aux patients.

Premièrement, les pénuries de médicaments peuvent avoir un impact direct sur les résultats thérapeutiques. Par exemple, les pénuries d'antinéoplasiques peuvent retarder le traitement, mener à l'utilisation de doses réduites ou nécessiter le recours à un traitement qui n'est pas fondé sur des données probantes, ce qui peut avoir des répercussions sur les taux de survie ${ }^{5}$. On a signalé également que les pénuries d'anesthésiants retardaient des opérations chirurgicales ou obligeaient l'emploi de médicaments de substitution associés à une prolongation de la convalescence ou à des nausées et à des vomissements postopératoires prolongés, ce qui mène potentiellement à de plus longs séjours hospitaliers et, globalement, à de moins bons résultats chirurgicaux $^{6}$. Les pénuries de norépinéphrine dans des hôpitaux des États-Unis ont été associées à une augmentation des taux de mortalité chez les patients subissant un choc septique'. L'Institute for Safe Medication Practices (États-Unis) a signalé un décès ayant été partiellement attribué au retard du traitement au bicarbonate de sodium d'un sepsis lié à l'acidose pendant une pénurie récente ${ }^{8}$.

Deuxièmement, l'emploi de médicaments moins connus ou dont la puissance, les concentrations et la forme pharmaceutique ont été modifiées, ce qui peut se produire lors d'une pénurie, augmentent les risques d'erreur médicamenteuse ${ }^{9}$. Les sphères de risque particulier ont été mises en lumière par l'Institut pour l'utilisation sécuritaire des médicaments du Canada (ISMP Canada $)^{9}$. Par exemple, le recours à des médicaments, à des concentrations, à des puissances ou à des formes pharmaceutiques autres fait apparaître un potentiel d'erreur dans l'ensemble du système d'utilisation des médicaments, que ce soit lors de la prescription, de la préparation, de l'administration ou du processus de suivi ${ }^{9}$. De plus, ces traitements de substitution peuvent ne pas apparaître dans les ressources habituelles de l'hôpital, comme les ensembles de modèles d'ordonnances, les manuels sur les médicaments parentéraux ou les bibliothèques de pompes intelligentes ${ }^{9}$. Deux sondages soumis à des membres du personnel en santé aux États-Unis ont mis en lumière ce risque. Parmi les 300 membres ayant répondu au sondage de 2017, et dont près des deux tiers étaient pharmaciens, $21 \%$ des répondants ont signalé au moins une erreur, et parmi les erreurs déclarées, plusieurs mettaient en cause des médicaments associés à un risque élevé, comme les opioïdes, le chlorure de potassium et l'épinéphrine ${ }^{8}$. Dans le sondage précédent, mené en 2010 , 
$35 \%$ des 1800 répondants ont déclaré au moins un incident évité de justesse ${ }^{10}$. ISMP Canada a signalé un accident lié à la pénurie d'épinéphrine topique, laquelle a été substituée par de l'épinéphrine parentérale 1:1000 pour une anesthésie locale; le mauvais médicament a été placé dans une seringue sans étiquette, puis administré par injection dans le site opératoire, ce qui a mené à un arrêt cardiaque puis au décès ${ }^{11}$.

Troisièmement, les pénuries de médicaments ont aussi des effets indirects sur la sécurité des patients. Dans le même sondage réalisé aux États-Unis, mentionné ci-dessus ${ }^{8}$, plus du tiers des répondants ont écrit des commentaires à propos des ressources humaines et financières nécessaires à la gestion des pénuries de médicaments. Ils ont indiqué plus particulièrement que le temps passé à s'occuper des pénuries de médicaments leur enlevait le temps qu'ils consacraient normalement aux soins des patients et aux activités portant sur la sécurité des médicaments, ce qui menait à des risques d'erreur plus importants ${ }^{8}$. Cette augmentation du risque d'erreur pourrait, en partie, être la conséquence de l'effet que les pénuries de médicaments peuvent avoir sur la charge de travail, la fatigue et les distractions mentales du personnel de première ligne? ${ }^{9}$. Les pharmaciens et les techniciens en pharmacie ont aussi indiqué que d'autres professionnels de la santé exprimaient souvent directement au personnel de pharmacie leur frustration à propos des pénuries de médicaments, ce qui minait le moral et augmentait encore davantage les risques d'erreur $^{8}$.

Bref, pour plusieurs raisons, les pénuries de médicaments représentent un risque pour les patients. Les pharmaciens et les techniciens en pharmacie peuvent néanmoins se sentir fiers de leur travail quotidien pour réduire ce risque. En effet, le faible nombre de résultats thérapeutiques négatifs graves signalés témoigne de l'importance du travail du personnel de la pharmacie, qui est aux premières lignes de la sécurité des patients. Or il est facile de relâcher son attention lorsqu'on fait face à un problème permanent qui nécessite une vigilance constante. Compte tenu de leur potentiel de nuisance pour les patients, les pénuries de médicaments sont manifestement plus qu'un simple bruit de fond. Nous devons garder cela à l'esprit et travailler à des solutions permanentes pour régler ce problème.

[Traduction par l'éditeur]

\section{References}

1. Vaillancourt R. Pénuries de médicaments : Que peuvent faire les pharmaciens d'hôpitaux? [éditorial]. Can J Hosp Pharm. 2012;65(3):178-9.

2. Videau M, Chemali L, Stucki C, Saavedra-Mitjans M, Largana S, Guerin A, et al. Drug shortages in Canada and selected European countries: a cross-sectional, institution-level comparison. Can J Hosp Pharm. 2019; 72(1):7-15.
3. Pénuries de médicaments [site Web]. Ottawa $(\mathrm{ON})$ : Pénuries de médicaments Canada. Publié au : https://www.penuriesdemedicamentscanada.ca/. Consulté le 13 novembre 2018.

4. Rinaldi F, de Denus S, Nguyen A, Nattel S, Bussières JF. Drug shortages: patients and health care providers are all drawing the short straw. Can J Cardiol. 2017;33(2):283-6.

5. McBride A, Holle LM, Westendorf C, Sidebottom M, Griffith N, Muller RJ, et al. National survey of drug shortages on cancer care. Am J Health Syst Pharm. 2013;70(7):609-17.

6. Hall R, Bryson GL, Flowerdew G, Neilpovitz D, Grabowski-Comeau, Turgeon AF; Canadian Perioperative Anesthesia Clinical Trials Group. Drug shortages in Canadian anesthesia: a national survey. Can J Anesth. 2013; 60(6):539-51.

7. Vail E, Gershengorn HB, Hua M, Walkey AJ, Rubenfeld G, Wunsch H. Association between US norepinephrine shortage and mortality among patients with septic shock. JAMA. 2017;317(14):1433-42.

8. Drug shortages continue to compromise patient care. Horsham (PA): Institute for Safe Medication Practices; 2018. Publié au : https:// www.ismp.org/resources/drug-shortages-continue-compromise-patient-care. Consulté le 9 janvier 2019.

9. Préoccupations quant à l'utilisation sécuritaire des médicaments en contexte de pénuries. ISMP Can Saf Bull. 2012;12(3):1-4. Publié au : https:// www.ismp-canada.org/fr/dossiers/bulletins/BISMPC2012-03.pdf. Consulté le 7 janvier 2019.

10. Special issue: Drug shortages: national survey reveals high level of frustration, low level of safety. Horsham (PA): Institute for Safe Medication Practices; 2010. Publié au : https://www.ismp.org/resources/special-issue-drug-shortagesnational-survey-reveals-high-level-frustration-low-level. Consulté le 23 janvier 2019.

11. Alerte: une pénurie d'épinéphrine topique à $1: 1000$ présente un risque pour la sécurité. Bull ISMP Can. 2015;15(3):1-3. Publié au : https://www. ismpcanada.org/fr/dossiers/bulletins/2015/BISMPC2015-03_Penurie EpinephrineTopique.pdf. Consulté le 13 janvier 2019.

Susan K Bowles, Pharm. D., M. Sc., travaille au service de pharmacie de la Régie de la santé de la Nouvelle-Écosse et à la Faculté de pharmacie de I'Université Dalhousie, à Halifax, en Nouvelle-Écosse. Elle est également rédactrice adjointe du Journal canadien de la pharmacie hospitalière.

Intérêts concurrents : Aucun déclaré.

Adresse de correspondance :

De Susan K Bowles

Pharmacie

Régie de la santé de la Nouvelle-Écosse

1796 rue Summer

Halifax NS B3H 3A7

Courriel : susan.bowles@nshealth.ca 